\title{
Bioactivities and protective effects of flavonoid pectolinarin in the management of hepatocellular carcinoma: Medicinal importance and therapeutic benefit in the medicine
}

\author{
Dinesh Kumar PATEL*, Kanika PATEL
}

Faculty of Health Sciences, Sam Higginbottom University of Agriculture, Technology and Sciences, Prayagraj, India

Introduction: Flavonoidal compounds have been used in the herbal medicine being as biomarkers for the chemical and pharmacological standardization of crude herbal plants material. Pectolinarin is important flavonoidal compound found to be present in the Cirsium japonicum.

Methods: To know the protective effects of pectolinarin in hepatocellular carcinoma, numerous experimental research work data have been collected and analyzed in the present investigation through literature databases. In order to understand the beneficial health aspect of pectolinarin in hepatocellular carcinoma, different modes of analysis of scientific data have been used in the present investigation including molecular mechanistic study through different literature databases. Pharmacological activities of pectolinarin have been investigated through literature data analysis to know the therapeutic potential of pectolinarin for the treatment of various forms of cancerous disorders including Hepatocellular carcinoma.

Results: From the literature data analysis of various scientific research works, it was found that pectolinarin revealed the beneficial effects on various form of human liver disorders and showed protective effects against Hepatocellular carcinoma. Literature data analysis revealed the pharmacological importance and therapeutic benefit of pectolinarin in the various form of cancerous disorders. Role of pectolinarigenin in treatment of hepatocellular carcinoma have been studied and found to be effective as it showed significant inhibitory potential on cell proliferation, migration and invasive abilities in the scientific research work.

Conclusions: Literature data analysis of various scientific research work revealed the beneficial effects of pectolinarin in hepatocellular carcinoma which could be used for the treatment of various forms of cancerous disorders. 